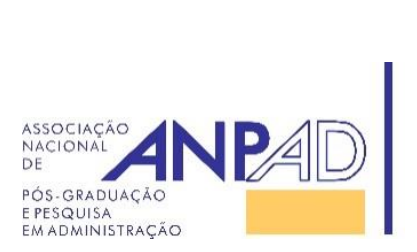

Disponível em

http://www.anpad.org.br/rac

RAC, Rio de Janeiro, v. 20, n. 3, art. 1, pp. 261-282, Maio/Jun. 2016 http://dx.doi.org/10.1590/1982-7849rac2016140048

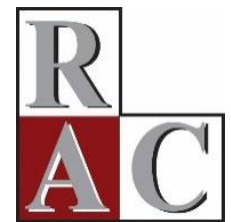

\title{
Mozart, Rock e a Ativação da Criatividade
}

\author{
Mozart, Rock and Creativity Activation
}

Guilherme Macedo de Souza Tieppo ${ }^{1}$

Germano Glufke Reis ${ }^{1,2}$

Djair Picchiai $^{1,3}$

Fundação Getulio Vargas ${ }^{1}$

Faculdades Metropolitanas Unidas - Programa de Pós-Graduação em Administração²

Faculdade Campo Limpo Paulista ${ }^{3}$

Artigo recebido em 22.09.2014. Última versão recebida em 02.06.2015. Aprovado em 03.06.2015. Publicado online em 01.12.2015. 


\title{
Resumo
}

A criatividade e o pensamento divergente são processos cognitivos envolvidos na geração de ideias e soluções novas, que podem contribuir com a inovação nas organizações. Os estudos sobre os antecedentes da criatividade têm focado características individuais, configuração de times e condições organizacionais; menos atenção tem sido dada à estimulação cognitiva. Nessa linha, o presente estudo investiga o efeito da estimulação cognitiva por meio da música sobre o pensamento divergente. Ele baseia-se nos estudos relacionados ao Efeito Mozart, que levaram à proposição de que a exposição à música pode induzir a um melhor desempenho, no curto prazo, em habilidades cognitivas específicas. Foi realizado um experimento com 133 estudantes de cursos de administração e de relações internacionais, divididos em diferentes grupos: dois foram expostos a estimulações musicais com estilos distintos (rock e Mozart) e outro não teve estimulação cognitiva antes da realização de tarefas criativas. O pensamento divergente foi avaliado por meio de uma abordagem subjetiva (método top 2 scoring). Observou-se, empregando a análise de variância (ANOVA), que os grupos expostos à música apresentaram maiores níveis de pensamento divergente, em comparação ao grupo controle. Implicações e limitações do estudo são discutidas.

Palavras-chave: criatividade; pensamento divergente; música; Mozart; estímulo cognitivo.

\begin{abstract}
Creativity and divergent thinking are cognitive processes involved in generation of new ideas and solutions that can contribute to innovation in organizations. Studies about creativity's antecedent have mostly focused on individual' characteristics, teams and organizational setting conditions; less attention has been given to cognitive stimulation. Along these lines, the present study investigates the effect of cognitive stimulation through music on divergent thinking. It is based on studies related to the Mozart effect that led to the proposition that exposure to music can lead to better performance in the short term, in specific cognitive abilities. An experiment with 133 students from business schools and international relations, divided into different groups was held: two were exposed to musical stimulation with different styles (rock and Mozart) and another had not cognitive stimulation before performing creative tasks. Divergent thinking was assessed by a subjective approach (top two scoring method). It was observed, using the variance analysis is (ANOVA), the groups exposed to music had higher levels of divergent thinking, compared to the control group. Implications and limitations of the study are discussed.
\end{abstract}

Key words: creativity; divergent thinking; music; Mozart; cognitive stimulation. 


\section{Introdução}

A inovação é peça-chave para que as empresas se tornem competitivas no longo prazo, pois permite às mesmas responderem e anteciparem novas demandas de mercados e clientes (Im, Montoya, \& Workman, 2013). Para que isto seja possível, é necessário que as organizações, times e pessoas tenham condições tanto de criar novas ideias quanto de colocá-las em prática. Nesse contexto, a criatividade é uma das dimensões que contribui com o processo de inovação, seja em produtos, seja em processos, organizacionais ou de marketing. Além disso, ela é considerada tanto um ingrediente importante quanto um dos primeiros passos para a inovação (Amabile, 1996; Anderson, Potočnik, \& Zhou, 2014; Dul \& Ceylan, 2014; Moon, 2013).

A capacidade de gerar múltiplas soluções novas e ideias criativas para um problema é o aspecto central do pensamento criativo (Gilhooly, Fioratou, Anthony, \& Wynn, 2007). Esta capacidade revela-se pela introdução de novidades adequadas a um público alvo através de outputs criativos (Dul \& Ceylan, 2014; Im et al., 2013), colocando em ação o pensamento divergente, que envolve justamente o processo individual de produção de ideias e soluções novas (Acar \& Runco, 2012; Plucker \& Renzulli, 1999; Woodman, Sawyer, \& Griffin, 1993). Contudo, apesar da relevância do tema, ainda se conhece pouco sobre as práticas que incrementam a geração de ideias novas nas organizações (Litchfield, 2008).

Conforme a revisão da literatura realizada por Anderson, Potočnik e Zhou (2014), estudos sobre os antecedentes da criatividade têm explorado, principalmente: as características das pessoas (personalidade, estilo cognitivo, competências, estados emocionais, etc.), as características das tarefas (complexidade, objetivos, etc.), o contexto social (liderança, influência dos clientes, redes sociais, etc.), as características dos times de trabalho (estrutura, clima, processos, etc.) e as condições organizacionais (iniciativas de recursos humanos, impactos da estratégia/estrutura, cultura, utilização de conhecimentos e recursos, etc.). Contudo, um tópico que poderia contribuir com esse campo, merecendo um exame mais detalhado, é o da estimulação cognitiva e o modo como ela impacta a criatividade e, mais especificamente, o pensamento divergente. De fato, embora os estados psicológicos e de humor relacionados à criatividade já venham sendo investigados (Anderson et al., 2014; Baas, De Dreu, \& Nijstad, 2008; Davis, 2009), os efeitos de estímulos cognitivos específicos sobre o pensamento divergente têm sido menos explorados.

Os pronunciados avanços recentes das neurociências, principalmente devido aos estudos que utilizam sistematicamente a ressonância magnética, têm lançado luz sobre o tema, evidenciando o modo como a estimulação induz alterações na atividade cerebral. Por exemplo, Fink et al. (2010) constataram que a estimulação cognitiva promovida pela exposição às ideias de outras pessoas pode promover incrementos no desempenho criativo; eles identificaram também, por meio de imagens de ressonância magnética, que esse efeito é acompanhado pela ativação de áreas do cérebro que estão associadas à integração semântica, à recuperação de memórias e a processos ligados à atenção. Wei et al. (2014), por sua vez, encontraram que a estimulação cognitiva, por meio de exercícios de pensamento divergente tal como solicitar, por exemplo, que os sujeitos proponham usos incomuns para objetos do dia a dia, tal como um guarda-chuva - promoveu incrementos na criatividade dos sujeitos do estudo relacionados à intensificação da conectividade funcional cerebral em repouso, entre determinadas regiões do cérebro (Wei et al., 2014). Assim, os conhecimentos gerados pelas neurociências e ciências cognitivas podem oferecer uma significativa contribuição interdisciplinar ao campo da administração de empresas (McDonald \& Tang, 2014), abrindo novas frentes para a pesquisa sobre os antecedentes do pensamento criativo nas organizações.

Os efeitos da estimulação por meio da música, foco deste estudo, têm merecido sistemática atenção na literatura científica; ainda há, contudo, pouca pesquisa e discussão sobre os efeitos da música especificamente no contexto organizacional (Prichard, Korczynski, \& Elmes, 2007; Styhre, 2013).

Observa-se que, na exposição à música, diferentes regiões do cérebro podem ser ativadas, relacionadas a processos cognitivos e emocionais (Peretz \& Zatorre, 2005). Popescu, Otsuka e Ioannides (2004), por exemplo, identificaram atividade em áreas motoras que variaram em função do ritmo da 
música. Por outro lado, a música também pode induzir alterações de humor: observa-se que músicas em tom maior, por exemplo, relacionam-se a emoções mais positivas do que as em tom menor (Sutton \& Lowis, 2008); de modo análogo, em outro estudo, observou-se que tonalidades maiores e com andamentos mais rápidos são mais relacionadas a sentimentos de alegria (Webster \& Weir, 2005). Reações emocionais e mudanças de humor em função da música são acompanhadas de atividade cerebral, envolvendo regiões do sistema límbico, ligadas às emoções (Särkämö et al., 2008). Além disso, há evidências de que a exposição continuada à prática musical possa induzir até mesmo um rearranjo de conexões cerebrais duradouro, com o passar do tempo (Gaser \& Shlaug, 2003; Schmithorst \& Holland, 2003).

Um estudo clássico nesse campo foi o conduzido por Rauscher, Shaw e Ky (1993, 1995), que propagou a ideia de efeito Mozart. De acordo com esta ideia, a exposição à música clássica - em particular, a trechos específicos das composições do músico Mozart (tal como da sonata K488), utilizados em vários experimentos - tende a promover efeitos cognitivos positivos. Desde então têm sido reportados, na literatura, resultados positivos associados à estimulação pela música: no desempenho em testes de inteligência espacial e temporal (Rauscher \& Shaw, 1998), na aprendizagem de tarefas de rotação espacial (Jausovec \& Habe, 2005), na solução de problemas de matemática (Pavlygina, Karamysheva, Tutushkina, Sakharov, \& Davydov, 2013), no tratamento de condições neurológicas (Kuester, Rios, Ortiz, \& Miranda, 2010), entre outros. Este trabalho contribui com a literatura sobre criatividade nas organizações ao lançar luz sobre a importância da estimulação cognitiva e, também, sobre as possíveis contribuições das neurociências e ciências cognitivas para a compreensão do tema, inspirando novas abordagens e caminhos de pesquisa. Ele também contribui investigando os efeitos da música clássica sobre o pensamento divergente, por meio de um experimento com estudantes universitários. Além disso, o trabalho amplia o escopo dos estudos supracitados, ao estudar o efeito de outro gênero musical, o rock, comparando-o tanto com a música clássica como com a ausência de estímulo específico.

\section{Música, Cérebro e o 'Efeito Mozart'}

Quando uma pessoa ouve música, a resposta do cérebro envolve um grande número de regiões, inclusive fora do córtex auditivo (Weinberger, 2004). De fato, as ondas de som que chegam aos ouvidos humanos são transmitidas ao cérebro pelo nervo auditivo, e ativam diferentes áreas como o córtex motor e o cerebelo. Logo, em função dessa amplitude de efeitos, é de se esperar que a música ajude na realização de tarefas não necessariamente musicais. Esse é o ponto de partida da observação experimental de que a música pode facilitar o processo de solução de problemas matemáticos (Pavlygina et al., 2013).

Efeito Mozart é a denominação dada à proposição - baseada em resultados de experimentos segundo a qual trechos específicos da música composta por Mozart (tal como da sonata K488) ativariam diferentes áreas do cérebro, podendo contribuir positivamente com a realização de atividades variadas no curto prazo, como em testes de raciocínio espacial (Rauscher, Shaw, \& Ky, 1993, 1995). Ao final dos anos 1980, neurocientistas tentaram, pela primeira vez, modelar a atividade cerebral em um computador na Universidade de Califórnia, quando foram percebidos certos padrões e ritmos na atividade das células nervosas. Ao invés de realizar uma impressão convencional, eles resolveram transformar essas ondas cerebrais em sons. Os resultados foram estimulantes: mapeamentos diferentes dos padrões encontrados resultavam em diferentes estilos musicais reconhecíveis. Deste modo, os neurocientistas concluíram que poderiam utilizar a música como uma janela para estimular funções cerebrais superiores (Leng \& Shaw, 1991). Surgiu, então, a ideia de se verificar a resposta do cérebro aos estímulos musicais, pois, já que a atividade cerebral podia soar como música, as ondas cerebrais poderiam, de maneira inversa, ser estimuladas por certos padrões musicais. Pelo fato de Mozart ter iniciado a compor em idade bastante baixa (aproximadamente quatro anos), e por haver registros de que elaborava músicas inteiramente compostas em sua mente, os pesquisadores optaram por realizar o experimento com uma de suas composições. A escolha da Sonata em D Maior decorreu de sua extrema 
organização, e foi utilizado o primeiro movimento (Allegro con spirito), de andamento acelerado (Leng \& Shaw, 1991).

A partir dessas constatações, em 1993 foi realizado um estudo piloto com estudantes, que deu origem à ideia de efeito Mozart (Rauscher et al., 1993). No experimento, três grupos de estudantes universitários responderam ao teste de inteligência de Stanford-Binet. O primeiro grupo ouviu, previamente, dez minutos da Sonata em D Maior para dois Pianos K488; o segundo grupo participou de uma sessão de relaxamento, com a mesma duração; finalmente, o terceiro grupo ficou dez minutos em silêncio. Os resultados apontaram que os alunos que ouviram a Sonata de Mozart antes do teste tiveram melhorias significativas, embora temporárias, na dimensão raciocínio espacial-temporal (Rauscher et al., 1993), resultado reforçado por estudo posterior (Rauscher et al., 1995). A duração do efeito da música, neste caso, é de curto prazo; ou seja, tende a durar de 10 a 15 minutos em uma exposição pontual ao estímulo musical (Nantais \& Schellenberg, 1999).

Por outro lado, outros estímulos, em paralelo à sonata de Mozart, também foram testados. Ratos em labirintos, por exemplo, expostos a essa estimulação, apresentaram maior rapidez de saída do labirinto, em comparação à música minimalista, que é um estilo caracterizado por uma estrutura cíclica marcada por padrões simplificados e repetição, durante um longo período, com pequenas variações do tema central da música (Obendorf, 2009); o mesmo ocorreu em comparação ao ruído branco (ruído produzido pela fusão de sons de todas as frequências que, em baixo volume, é utilizado como um som com características relaxantes) (Rauscher, Robinson, \& Jens, 1998). A estimulação com Mozart também foi comparada a Schubert (Nantais \& Schellenberg, 1999), quando se encontrou um efeito positivo similar em ambas as estimulações musicais. No mesmo experimento também foi analisado o impacto de ouvir histórias; nessa comparação, a preferência pessoal moderou os resultados: aqueles que preferiam histórias tiveram aumentos nos resultados, enquanto quem preferia música teve desempenho melhor ao ouvir Mozart.

Os experimentos apresentados acima sugerem que a música de Mozart influencia o desempenho em testes cognitivos, e constituem a base para os objetivos do presente estudo. Contudo, deve-se observar que tais experimentos abordaram apenas habilidades específicas, como lógica e associação, mas não se concentraram nas implicações para a criatividade e não foram estendidos para os efeitos de diferentes gêneros musicais sobre o pensamento divergente.

Por outro lado, para que se compreenda a relação entre a música e o pensamento divergente, é preciso, inicialmente, examinar o que explica a influência da música sobre o desempenho em testes cognitivos; evidências sugerem que fatores como excitação, humor e prazer gerados pela música são relevantes nessa influência. Estes aspectos - bem como outros fatores que associam a música à criatividade - são discutidos nos próximos tópicos.

\section{Críticas ao Efeito Mozart e o Papel da Excitação, do Humor e do Prazer}

Os experimentos de Rauscher e colegas foram replicados diversas vezes, encontrando, em geral, evidências que dão suporte às hipóteses do efeito Mozart; contudo, algumas replicações não alcançaram os mesmos resultados dos trabalhos originais, mencionados acima. Um exemplo foi a tentativa de Steele, Bass e Crook (1999), na qual três grupos foram expostos, respectivamente, a Mozart, música minimalista (de Phillip Glass) e silêncio. Os estímulos e instrumentos foram similares aos utilizados por Rauscher et al. (1995). Entretanto, os resultados obtidos foram diferentes, uma vez que a exposição a Mozart teve incrementos menores do que os alcançados com a estimulação por meio de música minimalista. Além disso, o estudo com Schubert versus Mozart (Nantais \& Schellenberg, 1999) não apontou diferenças significativas entre essas estimulações; ou seja, outros tipos de música podem, também, influenciar o desempenho cognitivo de curto prazo. De fato, com base nos estudos realizados, Thompson, Schellenberg e Husain (2001) propuseram que a exposição a diferentes tipos de música, não apenas a Mozart, pode melhorar o desempenho em determinados testes cognitivos, independentemente do 
estilo musical a que cada grupo foi exposto. Assim, em função das contradições identificadas nos estudos de efeito Mozart, outra abordagem foi proposta por Thompson et al. (2001), lançando a hipótese de que a música de Mozart (ou de Albinoni ou Schubert) em si não era a responsável por melhorias de desempenho cognitivo de curto prazo. De acordo com os autores, a melhoria de desempenho seria decorrente da excitação e das modificações de humor provocadas pela música.

Para medir a influência da excitação e do humor na realização de um teste de inteligência (teste Stanford-Binet), foi realizado um experimento com estudantes, submetidos a diferentes estímulos, que teriam o objetivo de aumentar e diminuir a excitação dos participantes (Thompson, Schellenberg, \& Husain, 2001). Um dos estímulos foi novamente a sonata de Mozart, e o outro consistiu em dez minutos de Adagio em G Menor para Órgão e Cordas, de Albinoni. A sonata de Mozart possui andamento acelerado e em tonalidade maior, e deveria, portanto, aumentar a excitação dos ouvintes (Thompson et al., 2001). A peça de Albinoni, por sua vez, é mais lenta e em tom menor, e deveria causar baixa excitação. Como medida complementar, cada um dos estudantes foi submetido a um teste que mede o nível de excitação e humor do indivíduo. Além disso, os participantes avaliaram o quanto gostaram da música. Os resultados indicaram que o desempenho do grupo que escutou Mozart foi melhor, de maneira significativa, na semana em que foram expostos à sonata, em comparação ao silêncio. O efeito foi contrário para o grupo que ouviu Albinoni. Não houve diferenças entre os escores do grupo de Mozart e Albinoni quando expostos ao silêncio; porém, quando expostos à música, o grupo que ouviu a sonata de Mozart apresentou desempenho expressivamente melhor (Thompson et al., 2001). Por outro lado, observou-se maior excitação e humor mais positivo no grupo que ouviu Mozart, em comparação ao grupo de Albinoni. Além disso, o grupo de Mozart também apresentou maior satisfação com a música do que o outro grupo. Essa constatação sugere que o Efeito Mozart, e os resultados encontrados, podem ser explicados pelo nível de excitação fomentado nos participantes e, também, pelo quanto apreciam a música ouvida (Thompson et al., 2001).

Outros experimentos foram realizados para sustentar esta hipótese. Em um deles, foram recrutadas 8.120 crianças do Reino Unido, de dez a onze anos, para a realização de um teste de habilidades espaciais, novamente com diferentes estímulos prévios. Um dos grupos ouviu música pop contemporânea (tal como a banda inglesa Blur). O segundo grupo ouviu Mozart, e o terceiro grupo ouviu uma discussão do experimento entre o autor e um jornalista. $\mathrm{O}$ resultado mostrou desempenho superior entre as crianças que ouviram música pop. Não houve diferença entre os resultados dos demais estímulos. Por meio do que chamaram de o efeito Blur, os pesquisadores concluíram que o desempenho em testes espaciais é melhor ao se escutar músicas que geram prazer para o ouvinte (Schellenberg \& Hallam, 2005), tal como a música mais popular da época para as crianças da amostra (neste caso, da banda Blur).

Estes estudos expressam uma nova forma de explicação para a influência da música sobre testes cognitivos, neste caso ainda mais acessível do que as teorias que se apoiam especificamente na música de Mozart, já que a hipótese emergente propõe que melhorias no desempenho cognitivo se associam à música que gera prazer no ouvinte. Assim, dimensões como excitação, prazer, estados emocionais e de humor têm um papel relevante na estimulação cognitiva por meio da música.

A grande maioria dos trabalhos, contudo, examina basicamente a influência da música sobre o desempenho cognitivo, tal como a inteligência espaço-temporal. Assim, é relevante que outros processos cognitivos, menos abordados, também sejam explorados. É o caso da criatividade, investigada no presente trabalho.

\section{Criatividade: Qual é o Efeito da Música?}

Por um lado, pesquisas na área das neurociências têm reforçado que a música influencia as emoções das pessoas. Por exemplo, ela ativa circuitos cerebrais envolvidos nas sensações de prazer e recompensa (Blood \& Zatorre, 2001). Tais áreas do cérebro são as mesmas que respondem a estímulos 
indutores de sensações de euforia e relacionados à sobrevivência (alimentos, sexo, etc.) (Blood \& Zatorre, 2001). A música promove atividade em áreas do cérebro que são chave para os processos emocionais (amígdala, hipotálamo, etc.) (Koelsch, 2014).

Por outro lado, estudos sobre criatividade nas organizações têm mostrado que a presença de emoções positivas e prazer favorecem a ativação e expressão da criatividade. De fato, a presença de afetos positivos (tais como prazer, satisfação com o grupo, alegria, etc.) tende a facilitar a emergência da variação cognitiva, representada por novas combinações de ideias, conceitos e conhecimentos, gerando novas associações e induzindo à criatividade (Amabile, Barsade, Mueller, \& Staw, 2005). Além disso, condições organizacionais, tais como o perfil da liderança e um ambiente seguro, podem desencadear um sentimento de energia e o nível de vitalidade, que são pré-requisitos para catalisar a criatividade (Atwater \& Carmeli, 2009). O que se observa nesses estudos é que a atmosfera emocional, humor e prazer são elementos facilitadores na emergência do pensamento criativo. Desse modo, e considerando-se também a relação neurológica da música com as emoções, é de se esperar que o estímulo musical possa impactar positivamente a criatividade.

Em linha com a hipótese da excitação e humor gerados pela música mencionada na seção anterior, um estudo realizado com crianças japonesas de cinco anos de idade explorou os efeitos da música sobre a criatividade, empregando desenhos. Foram definidos quatro grupos, com diferentes estímulos musicais: um grupo ouviu Mozart, o segundo ouviu Albinoni, o terceiro ouviu canções familiares para crianças, enquanto o último grupo cantou canções familiares infantis, acompanhadas de um piano (Schellenberg, Nakata, Hunter, \& Tamoto, 2007). As crianças que ouviram e cantaram músicas infantis obtiveram avaliações superiores nos três critérios mensurados. Não houve diferenças entre cantar e escutar as canções infantis em termos de criatividade. Este estudo mais uma vez sugere que a excitação e o humor gerados através da música são os responsáveis pelo melhor desempenho, contribuindo para que se compreenda os resultados de experimentos que não deram suporte ao Efeito Mozart (Schellenberg et al., 2007). Além disso, este trabalho sugere que a capacidade criativa pode ser estimulada por meio da música, ainda mais se for levado em conta o fato de que as crianças não tinham conhecimento de que estavam sendo avaliadas. Outros estudos também compararam a ativação cerebral de músicos e não músicos, por meio de neuroimagem funcional e testes de pensamento divergente, e encontraram evidências que suportaram a hipótese do aumento da criatividade em músicos treinados, em relação a não músicos (Gibson, Folley, \& Park, 2009). O que se observa, nesses estudos, é que a atmosfera emocional, humor e prazer são elementos facilitadores na emergência do pensamento criativo. Desse modo, e considerando-se também a relação neurológica da música com as emoções, é de se esperar que o estímulo musical possa impactar positivamente a criatividade. Assim, pode-se propor a seguinte hipótese:

H1: a estimulação pela música de Mozart relaciona-se a maiores níveis de pensamento divergente, em comparação à ausência de estimulação musical.

Por outro lado, no presente estudo, optou-se pela inclusão de um segundo estilo musical, além de Mozart, em função dos argumentos apresentados no referencial teórico, que colocam que os componentes de excitação, variação de humor e prazer são aspectos centrais na estimulação promovida pela música (Chabris, 1999; Thompson et al., 2001). Além disso, outros gêneros musicais também podem produzir resultados similares aos da música de Mozart, podendo promover incrementos em diferentes aspectos cognitivos (Schellenberg et al., 2007); espera-se, então, que o mesmo efeito ocorra para o pensamento divergente.

A escolha do rock, como estilo a ser investigado, baseou-se no estudo de Schellenberg e Hallam (2005), que identificaram que determinadas músicas pop - prazerosas para os adolescentes que constituíam a amostra - promoveram melhorias de desempenho em testes espaciais. Assim, de modo análogo, o outro estilo musical testado neste trabalho foi um rock contemporâneo do gênero indie rock, estilo bastante presente nas rádios e muito popular na última década. Ainda, seu andamento acelerado e tonalidade maior possuem o potencial de estimular excitação e prazer entre os participantes da amostra. Com base nos argumentos de que os efeitos da música se aplicam a diferentes gêneros, pode-se propor que: 
H2: a estimulação por meio do rock relaciona-se a maiores níveis de pensamento divergente, em comparação à ausência de estimulação musical.

H3: não há diferenças entre os níveis de pensamento divergente, quando a estimulação por meio de Mozart e a estimulação por meio do rock são comparadas.

\section{Metodologia}

O experimento realizado neste trabalho testou se a exposição à música tem ou não um efeito positivo sobre a criatividade das pessoas; mais especificamente, é investigada a influência sobre o processo de pensamento divergente. Enquanto o pensamento convergente envolve processos lógicos para a identificação de uma única solução, ou da melhor solução para um dado problema (Acar \& Runco, 2012), o pensamento divergente, por sua vez, leva à geração de novas ideias e conexões por meio da exploração de múltiplas alternativas e soluções possíveis. Esse é um processo cognitivo envolvido na geração de ideias criativas e soluções novas e tem sido sistematicamente empregado na mensuração de diferenças individuais relacionadas à criatividade (Plucker \& Renzulli, 1999; Silvia, 2011; Silvia et al., 2008).

O pensamento divergente é bastante útil e satisfatoriamente confiável como medida para a criatividade. De fato, Runco e Acar (2012) colocam que os estudos que adotam índices de confiabilidade baseados na consistência entre itens têm alcançado, em sua maioria, coeficientes de confiabilidade acima de 0,70 (alfa de Cronbach), que são considerados adequados (Hair, Black, Babin, \& Anderson, 2010). Por outro lado, a análise da confiabilidade entre avaliadores é indicada quando há subjetividade, ainda que mínima, no processo de pontuação do desempenho dos sujeitos; nessa abordagem, os autores identificaram coeficientes iguais ou acima de 0,90 (Runco \& Acar, 2012). Benedek, Mühlmann, Jauk e Neubauer (2013), por sua vez, avaliaram a confiabilidade de uma abordagem subjetiva de aferição do pensamento divergente na qual os participantes devem escolher duas respostas mais criativas (método top 2 scoring), e encontraram, de modo geral, coeficientes acima de 0,80 (alfa de Cronbach) para a fluência e acima de 0,70 para a originalidade dos sujeitos, quando estes escolhem duas ou mais respostas que consideram as mais criativas. Do ponto de vista de validade, tem-se observado que o pensamento divergente pode ser discriminado de outros construtos relevantes para os estudos sobre criatividade, tais como inteligência geral (Runco \& Acar, 2012) e traços de personalidade (Silvia et al., 2008). Além disso, observou-se que mais da metade da variância em pensamento divergente pode ser explicada por características de personalidade e de estilo de vida, que são, teoricamente, antecedentes da criatividade; esse teste de validade apontou que os escores de pensamento divergente refletem o efeito esperado para o construto (Silvia et al., 2008).

As características da amostra, bem como detalhes sobre a mensuração do pensamento divergente, procedimentos do experimento e músicas adotadas, são descritos a seguir.

\section{Participantes}

Os participantes do experimento foram 133 estudantes universitários de 18 a 26 anos, que cursavam administração de empresas (86\%) e relações internacionais (14\%) em duas instituições de ensino que atendem alunos provenientes de classes sociais altas na cidade de São Paulo. A amostra foi constituída por conveniência, em instituições às quais os pesquisadores tiveram acesso; eles não eram professores dos sujeitos da amostra. As turmas foram distribuídas aleatoriamente, em três grupos, com estímulos distintos: Mozart, rock contemporâneo e grupo de controle.

Duas classes de alunos ouviram Mozart antes da avaliação de pensamento divergente, totalizando 62 alunos entre 18 e 26 anos ( $M=20,1$ anos, $D P=1,8)$. Para duas outras classes o estímulo utilizado foi o 
rock, totalizando 48 alunos entre 18 e 23 anos $(M=19,2$ anos, $\mathrm{DP}=0,9)$. $\mathrm{O}$ grupo de controle continha 23 alunos entre 18 e 22 anos $(\mathrm{M}=18,9$ anos, $\mathrm{DP}=1,1)$. A Tabela 1 mostra os detalhes de cada grupo.

Tabela 1

\section{Características Gerais da Amostra}

\begin{tabular}{cccccc}
\hline Estímulo & Amostra & Idade média & Desvio Padrão & Sexo M & Sexo F \\
\hline Mozart & 62 & 20,1 & 1,8 & $32(52 \%)$ & $30(48 \%)$ \\
Rock & 48 & 19,2 & 0,9 & $14(29 \%)$ & $34(71 \%)$ \\
Controle & 23 & 18,9 & 1,1 & $9(39 \%)$ & $14(61 \%)$ \\
\hline
\end{tabular}

\section{Mensuração do pensamento divergente}

A avaliação de pensamento divergente adotada foi proposta e testada por Silvia et al. (2008), como uma alternativa a alguns dos testes comumente utilizados para o pensamento divergente, cujos escores consideram apenas as respostas singulares dos avaliados; ou seja, eles consideram apenas as respostas únicas como respostas criativas. O Torrance Test of Creative Thinking (TTCT), por exemplo, atribui pontos para respostas que se encontrem fora de uma amostra normativa de respostas comuns (Torrance, 2008), e os pontos são somados para obter um índice de originalidade. A pontuação singular, contudo, possui limitações importantes (Benedek, Mühlmann, Jauk, \& Neubauer, 2013; Silvia, 2011; Silvia et al., 2008). Primeiro, a alta correlação (próxima a 0.90) entre os escores de singularidade (respostas únicas) e os escores de fluência (quantidade de respostas da pessoa): a chance de produzir uma resposta única aumenta, conforme aumenta o número total de respostas. Segundo, o tamanho da amostra influencia os escores de singularidade: aumentando a amostra, respostas criativas tornam-se menos frequentes (Silvia, Martin, \& Nusbaum, 2009). Terceiro, singularidade é um conceito ambíguo de filtragem difícil, pois não se pode dizer que respostas criativas sejam necessariamente aquelas únicas (que podem até mesmo ser bizarras ou inapropriadas), uma vez que elas devem também ser apropriadas para a tarefa (Silvia et al., 2008).

À luz destes argumentos, empregou-se, neste experimento, um processo de avaliação de pensamento divergente que utiliza um índice de classificação subjetivo das respostas (Silvia et al., 2008). Em linhas gerais, são apresentadas aos sujeitos tarefas de pensamento divergente. Nessas tarefas é solicitado aos participantes que produzam o máximo de respostas criativas possíveis (Plucker \& Renzulli, 1999) em um determinado período de tempo; os sujeitos também devem assinalar as duas respostam que avaliam como sendo as mais criativas (método top 2 scoring) (Silvia et al., 2008). A seguir, avaliadores analisam as respostas geradas, atribuindo-lhes escores a partir de critérios pré-definidos (explicados adiante). Esta abordagem apresenta algumas vantagens: considera o melhor desempenho que os participantes podem alcançar, torna constante o número de respostas pelos quais os sujeitos são avaliados, privilegia as melhores ideias em detrimento de um maior número de respostas.

A confiabilidade desta abordagem foi testada por meio do enfoque da generalizability theory (Cronbach, Gleser, Nanda, \& Rajaratman, 1972; Mushquash \& O'Connor, 2006). Foi identificado que tarefas/exercícios de pensamento divergente de usos incomuns e de instâncias (descritos abaixo) alcançam os índices de confiabilidade requeridos $(G>0,80)$ com apenas dois ou três avaliadores; exercícios de consequências alcançam os índices com quatro ou mais avaliadores. Com relação à validade, foi testada a relação do pensamento divergente com outros construtos que, teoricamente, são seus antecedentes: características de personalidade e estilo de vida. Nesse teste observou-se que mais da metade da variância em pensamento divergente pode ser explicada por essas variáveis. Assim, o teste de validade evidenciou que os escores de pensamento divergente refletiram o efeito esperado para o construto (Silvia et al., 2008).

Com relação às tarefas de pensamento divergente adotadas em estudos na área de criatividade, três tipos (conforme a sua natureza) são frequentemente utilizados (Silvia, 2011): (a) exercícios em que 
o participante deve elencar usos criativos para objetos comuns, tal como tijolos, latas, etc. (tarefas de usos incomuns); (b) tarefas em que o sujeito deve gerar exemplos criativos dentro de uma categoria comum, tal como a forma circular, ruído alto, um contexto específico, etc. (tarefas de instâncias, ou seja, de casos incomuns para uma dada categoria); (c) tarefas em que o participante deve propor implicações criativas para uma situação; por exemplo, consequências de não precisar mais dormir ou de as pessoas encolherem (tarefas de consequências).

Tal como Silvia et al. (2008), neste trabalho foi utilizada uma tarefa de cada um desses três tipos, para a avaliação do pensamento divergente dos participantes; são empregados exercícios de diferentes naturezas, pois eles abordam aspectos complementares no processo criativo (Silvia, 2011). O método de avaliação subjetiva não prescreve, contudo, quais são as tarefas específicas a serem administradas, viabilizando inúmeras variações (por exemplo: Benedek et al., 2013; Silvia, 2011; Silvia, Nusbaum, Berg, Martin, \& O’Connor, 2009); a ênfase da abordagem é sobre a forma de avaliação e sobre a utilização de top scores.

Os exercícios de usos incomuns e de consequências empregados neste estudo já são conhecidos, pois se baseiam em tarefas de pensamento divergente utilizadas em trabalhos pioneiros de Guilford e colaboradores (Christensen, Guilford, Merrifield, \& Wilson, 1960; Christensen, Merrifield, \& Guilford, 1958); em particular, as tarefas adotadas abordam os usos incomuns para um tijolo e as consequências de não precisar mais dormir, também empregadas por Silvia et al. (2008). Os enunciados desses exercícios foram traduzidos do inglês (Silvia et al., 2008), de modo consistente com definições em português sobre tarefas de pensamento divergente (Alencar \& Fleith, 2010). A tradução foi checada por meio da retradução para o inglês e da comparação entre a versão original e a retraduzida; avaliou-se que foram preservados o conteúdo e sentido originais.

Com relação à tarefa de instâncias, embora tenha sido preservada a sua natureza, optou-se por adotar o ambiente da sala de estar como categoria comum para as respostas criativas (ao invés de formas circulares, como em Silvia et al., 2008) (ver Tabela 2). Foi considerado que as outras tarefas já envolviam exercícios essencialmente abstratos e buscou-se favorecer o comprometimento dos participantes, alunos universitários, incluindo uma tarefa de cunho mais concreto.

Assim, a Tabela 2 apresenta os três exercícios empregados; para cada um deles, o participante deveria listar, em três minutos, uma série de respostas criativas. Ao final de cada exercício, os participantes deveriam circular as duas respostas que considerassem mais criativas. Posteriormente, as respostas foram analisadas por quatro avaliadores.

Tabela 2

Exercícios de Pensamento Divergente

Exercício 1 (usos incomuns) Nesta questão, você deve escrever todos os usos criativos e originais para um tijolo. Certamente, existem diversos usos comuns (como construir uma casa), mas para este exercício você deve enumerar os usos diferentes, não usuais em que consiga pensar. No final do exercício, circule os dois que julgar mais criativos.

Exercício 2 (instâncias) Nesta questão, você deve escrever todos os objetos, utensílios e coisas em geral que existem em uma Sala de Estar. Certamente, existem muitas coisas óbvias em uma sala de estar, mas para este exercício você deve apontar as criativas, não usuais, em que consiga pensar. No final do exercício, circule as duas que julgar mais criativas.

Exercício 3 (consequências) Para esta questão, imagine que as pessoas não precisam mais dormir. Quais seriam as consequências? Escreva todas as consequências originais e criativas em que consiga pensar. No final do exercício, circule as duas consequências que julgar mais criativas. 
Os avaliadores atribuíram a cada uma das respostas uma pontuação de 1 a 5 (1=nada criativa; $5=$ excepcionalmente criativa) (Silvia et al., 2008). Este escore de 1 a 5 atribuído às respostas considera três atributos: resposta incomum, associação remota e resposta inteligente/esperta (Wilson, Guilford, \& Christensen, 1953). Ideias criativas são pouco comuns, pois são aquelas que ocorrem com menor frequência na amostra. Ideias criativas também apresentam associação remota com eventos e objetos do dia a dia, saem do trivial. Por último, o terceiro critério avalia se a resposta é perspicaz, irônica, bemhumorada, esperta, tanto no conteúdo, como na forma de expressão da ideia; tais respostas tendem a ser mais criativas (Silvia et al., 2008). As dimensões são consideradas em conjunto, pois são correlacionadas e foram extraídas em um único fator (Wilson et al., 1953). Alguns critérios adicionais também são utilizados: no exercício 1 (usos incomuns), dá-se notas baixas para a utilização padrão do objeto (ex: construir casa); no exercício 2 (objetos), atribui-se pontuação baixa para objetos visíveis no local do experimento (ex: quadro negro).

A partir daí, duas médias são obtidas por pessoa, para cada exercício: uma média simples (Escore) de todas as respostas dadas pelo participante no exercício, e uma média das duas respostas escolhidas como as melhores do seu conjunto de respostas no exercício (TopEscore). Esta segunda média evita que indivíduos que enumeram um maior número de respostas, sendo algumas menos criativas, obtenham um escore menor, sendo penalizados em relação a participantes que enumeram poucas soluções (Benedek et al., 2013; Silvia et al., 2008).

A avaliação escolhida contorna as limitações da pontuação singular, apresentadas anteriormente. Além disso, uma vez que os participantes são induzidos a serem criativos, tende a estimular maior criatividade das respostas e um aumento na variância dos escores, permitindo que estes sejam indicadores mais válidos de diferenças individuais (Silvia et al., 2008).

Foi analisada, neste estudo, a confiabilidade da mensuração de pensamento divergente. Trata-se de uma estimativa da consistência dos escores, sendo que a consistência entre os avaliadores é a mais indicada para abordagens subjetivas (Runco \& Acar, 2012). Assim, o alfa de Cronbach foi calculado a partir das médias dos escores atribuídos por cada um dos avaliadores a cada pessoa. $\mathrm{O}$ alfa para o indicador Escore foi 0,79; para TopEscore, foi 0,72. Ambos podem ser considerados satisfatórios (Hair et al., 2010).

\section{Procedimentos}

Foram tomados cuidados com relação a aspectos éticos, baseados em recomendações da American Psychological Association (2002), para pesquisas com seres humanos, tais como: respeito aos participantes, estes foram informados sobre os objetivos e passos do experimento, a participação foi voluntária, as respostas foram tratadas de forma a garantir sigilo aos sujeitos.

Os procedimentos para a mensuração do pensamento divergente foram análogos aos da pesquisa original (Silvia et al., 2008), que também envolveu estudantes universitários em sala de aula. Contudo, pequenas adaptações foram realizadas com vistas a acomodar o design do experimento (três grupos com estimulações musicais distintas), o número maior de participantes e, também, para incluir a estimulação musical antes da avaliação de pensamento divergente. Os procedimentos foram padronizados em todos os grupos.

A avaliação de pensamento divergente foi administrada em diferentes salas de aula, no início das respectivas aulas. A determinação do estímulo para cada uma das classes foi aleatória. Inicialmente foi explicado aos alunos - por meio de instruções padronizadas - que eles iriam participar de um teste e que, antes de realizá-lo, iriam ouvir uma música de cinco minutos, o que seria uma preparação para a realização do mesmo (para o grupo de controle o teste foi aplicado sem estímulo e as instruções iniciais não fizeram menção à estimulação musical). Foi recomendado aos alunos que se concentrassem na música durante a sua exibição. Antes de iniciar o estímulo, cada aluno recebeu uma folha com os 
enunciados dos três exercícios de criatividade. As folhas foram distribuídas com o texto virado para baixo, de modo que os alunos não tiveram acesso a ele até o início do teste.

O estímulo escolhido de Mozart foi a Sonata em D Maior para dois Pianos, K488, a mesma utilizada no experimento de Shaw e Rauscher em 1993 e em diversos experimentos posteriores. Foi utilizado o mesmo trecho, o movimento 1 (Allegro con spirito), que apresenta andamento acelerado. No experimento original foi empregado um trecho de 10 minutos; dadas as restrições práticas para a realização deste estudo em salas de aula (de tempo, principalmente), foi utilizado um trecho de 5 minutos para a estimulação musical.

Com o objetivo de testar também a hipótese da excitação e humor e com base no estudo com música pop de Schellenberg e Hallam (2005), o outro estímulo musical escolhido foi um rock contemporâneo chamado In One Ear, da banda inglesa Cage the Elephant. Essa música foi lançada no ano de 2008, e seu gênero é definido como Indie Rock, estilo musical bastante influente na última década. O que motivou a escolha desta música, assim como em Schellenberg e Hallam (2005), foi o seu andamento acelerado e o seu estilo musical condizente com a música popular atual, reproduzida nas rádios. Assim, a música escolhida apresentava potencial para produzir, ao mesmo tempo, prazer (entre os participantes, que são estudantes universitários) e excitação. Em relação ao grupo de controle, os exercícios foram distribuídos imediatamente, sem a necessidade do estímulo prévio.

A abordagem empregada não demanda que os avaliadores sejam experientes na avaliação do pensamento divergente (Silvia, Winterstein, \& Willse, 2008), mas requer que eles sejam treinados para a utilização dos critérios de avaliação. Assim, optou-se por compor um grupo de avaliadores que não tivessem, em sua maioria, repertório prévio no campo da criatividade (experiência com outros testes, conhecimento de outros conceitos e abordagens, etc.), facilitando o aprendizado do método adotado e prevenindo julgamentos pré-concebidos. Considerando-se os melhores índices de confiabilidade alcançados com três ou mais avaliadores (Silvia et al., 2008), foram envolvidos quatro avaliadores (dois administradores de empresa, um engenheiro e um psicólogo). Foram realizados treinamento e orientação sobre como avaliar as respostas, visando a redução do viés e das inconsistências entre os avaliadores. Também seguindo Silvia et al. (2008), eles tiveram acesso apenas a uma lista de todas as respostas tabuladas em uma planilha eletrônica, divididas entre os três exercícios. Portanto, não tinham conhecimento de qual estímulo (Mozart, rock, nenhum estímulo) era referente a qual resposta, evitando escores enviesados; ademais, três deles não eram pesquisadores envolvidos com o estudo e, portanto, não conheciam as hipóteses propostas. Além disso, as avaliações são individuais; não há discussão entre os avaliadores (Silvia et al., 2008). Conforme mencionado na seção anterior, foi alcançado um nível de confiabilidade satisfatório (alfa de Cronbach > 0,70).

\section{Resultados}

Ao todo, os 133 participantes geraram: 621 respostas para o exercício 1 (usos incomuns); 957 respostas para o exercício 2 (casos incomuns); 656 para o exercício 3 (consequências incomuns). Exemplos de respostas dos participantes podem ser observadas na Tabela 3: 
Tabela 3

\section{Exemplos de Respostas}

\begin{tabular}{ll}
\hline Exercício 1 (usos incomuns) & $\begin{array}{l}\text { Construir um muro, trave de gol, apoiar livros, quebrar e fazer um quebra- } \\
\text { cabeças, âncora de boia, uivar pelos buracos do tijolo, colocar sobre o pedal do } \\
\text { carro para checar as luzes traseiras, etc. }\end{array}$ \\
\hline Exercício 2 (instâncias) & $\begin{array}{l}\text { Abajur, TV, puffs, pernilongo, animais empalhados, pilhas AA perdidas, } \\
\text { uniforme militar enquadrado, pôsteres de filmes noir, mini cama elástica, } \\
\text { xilofone, etc. }\end{array}$ \\
\hline Exercício 3 (consequências) & $\begin{array}{l}\text { Ninguém mais sonharia, stress, evolução científica duas vezes mais rápida, boom } \\
\text { na economia mundial, olheiras, carga de trabalho aumentaria, travesseiros seriam } \\
\text { apenas e somente enfeites, etc. }\end{array}$ \\
\hline
\end{tabular}

Após a atribuição de pontuações pelos avaliadores, a análise dos resultados foi realizada em duas etapas. $\mathrm{O}$ indicador de criatividade que inclui todas as respostas de cada exercício foi denominado Escore e o indicador que considera apenas as duas melhores respostas de cada exercício (respostas circuladas pelos respondentes) foi denominado TopEscore. Em cada uma dessas etapas, foram comparadas as médias gerais dos exercícios do teste de criatividade (Escore geral e TopEscore geral), para determinar a sua relevância na pontuação total. São apresentados, a seguir, os resultados de cada uma das análises.

\section{Análise escore}

Na Tabela 4, podem ser verificados os resultados da análise das médias escore (M) e desvios padrões (DP) para cada grupo (controle, Mozart e rock), em cada exercício. A Tabela 4 apresenta também o Escore geral, que é uma média dos três exercícios.

Tabela 4

Estatística Descritiva para a Análise Escore

\begin{tabular}{lcccc} 
& Grupo & n & M & DP \\
\hline Escore exercício 1 & Controle & 23 & 2,08 & 0,35 \\
(usos incomuns) & Mozart & 62 & 2,22 & 0,38 \\
& Rock & 48 & 2,09 & 0,31 \\
\hline Escore exercício 2 & Controle & 23 & 1,84 & 0,51 \\
(casos incomuns) & Mozart & 61 & 2,15 & 0,57 \\
& Rock & 48 & 2,23 & 0,56 \\
\hline Escore exercício 3 & Controle & 23 & 2,30 & 0,21 \\
(conseq. incomuns) & Mozart & 62 & 2,51 & 0,34 \\
& Rock & 48 & 2,49 & 0,28 \\
\hline Escore geral & Controle & 23 & 2,06 & 0,24 \\
& Mozart & 62 & 2,29 & 0,28 \\
& Rock & 48 & 2,26 & 0,26 \\
\hline
\end{tabular}

O Escore geral é o indicador de maior interesse para esta análise, na mensuração das diferenças individuais relacionadas à criatividade (Silvia et al., 2008). Foi realizado, então, o teste da ANOVA, a fim de se comparar as médias dos grupos controle, Mozart e rock. Os testes dos pressupostos de 
normalidade e de igualdade de variâncias (testes Kolmogorov-Smirnov e Levene) não indicaram violação; no primeiro, o resultado foi $\mathrm{D}(133)=0,57, \mathrm{p}=0,20$, para o teste de Levene foi $\mathrm{F}(2,130)=$ $0,23, \mathrm{p}=0,80$.

O resultado da ANOVA rejeita a hipótese nula, em um nível de significância de 1\%, de que as médias são iguais $(F(2,130)=6,34, p=0,00)$. Assim, observa-se um efeito da estimulação cognitiva por meio da música, nesses escores de pensamento divergente.

A seguir, foi empregado o teste post hoc de Tukey, a fim de se examinar entre quais grupos ocorreram diferenças significantes entre as médias, empregando-se o indicador escore. Identificou-se que a diferença entre as médias dos grupos Mozart e controle é significante $(\mathrm{p}<0,01)$, sendo que estudantes expostos à música apresentaram maiores escores para pensamento criativo (ver Tabela 4), o que deu suporte à hipótese 1. Também foi observada uma diferença significante ente os grupos rock e controle ( $\mathrm{p}<0,01)$; novamente os escores para criatividade foram maiores em função da exposição à música, dando suporte à hipótese 2. Por outro lado, a diferença entre Mozart e rock não foi significante $(\mathrm{p}>0,05)$, o que deu suporte à hipótese 3 .

\section{Análise topescore}

A análise TopEscore é ainda de maior interesse, já que considera apenas as duas melhores respostas de cada indivíduo, em cada exercício. Uma vez que a média é gerada com um número fixo de respostas - potencialmente as de maior escore - é possível contornar a penalização atribuída àqueles que produzem mais respostas no exercício. De outro modo, essa penalização poderia ocorrer, já que uma quantidade maior de respostas tende a aumentar o número de respostas menos criativas (e com pontuações menores), reduzindo a média.

Da mesma forma que na seção anterior, a Tabela 5, a seguir, mostra a estatística descritiva de cada um dos grupos, nos três exercícios realizados. Além dos escores médios (M) e desvios-padrões (DP) de cada exercício, a tabela apresenta o TopEscore geral: as médias totais para a mensuração do pensamento divergente.

Tabela 5

Estatística Descritiva para a Análise TopEscore

\begin{tabular}{lcccc}
\cline { 2 - 4 } & Grupo & n & M & DP \\
\hline Escore exercício 1 & Controle & 23 & 2,22 & 0,44 \\
(usos incomuns) & Mozart & 62 & 2,46 & 0,58 \\
& Rock & 48 & 2,33 & 0,49 \\
\hline Escore exercício 2 & Controle & 23 & 2,17 & 0,62 \\
(casos incomuns) & Mozart & 62 & 2,50 & 0,60 \\
& Rock & 48 & 2,46 & 0,60 \\
\hline Escore exercício 3 & Controle & 23 & 2,40 & 0,26 \\
(conseq. incomuns) & Mozart & 62 & 2,66 & 0,47 \\
& Rock & 48 & 2,65 & 0,43 \\
\hline Top Escore geral & Controle & 23 & 2,25 & 0,28 \\
& Mozart & 62 & 2,53 & 0,37 \\
& Rock & 48 & 2,47 & 0,35 \\
\hline
\end{tabular}


Também foi realizado o teste ANOVA para o indicador TopEscore geral. Observou-se que, neste caso, foi atendido o pressuposto de igualdade de variâncias, $\operatorname{com} F(2,130)=1,56, p=0,22$, mas não o de normalidade, com $\mathrm{D}(133)=0,09, \mathrm{p}=0,01$. Assim, para fins de verificação das comparações entre as médias dos grupos controle, Mozart e rock foi realizado, além da ANOVA, um teste não paramétrico indicado para a comparação de médias entre amostras/grupos independentes, o teste de Mann-Whitney. Esse teste não adota pressupostos, não demandando a verificação de normalidade e/ou igualdade de variâncias (Siegel \& Castellan,1988).

Com relação à ANOVA, considerando-se um índice de significância de 1\%, observou-se uma diferença significante entre as médias $(\mathrm{F}(2,130)=5,38, \mathrm{p}=0,00)$. Assim, os resultados indicam haver diferença entre as médias dos grupos de estímulo no teste de criatividade, rejeitando-se a hipótese nula. O teste post hoc de Tukey foi realizado a fim de se examinar entre quais grupos ocorreram diferenças significantes entre as médias. Identificou-se que a diferença entre as médias dos grupos Mozart e controle é significante $(\mathrm{p}<0,01)$, sendo que estudantes expostos à música apresentaram maiores escores para pensamento criativo (ver Tabela 5), o que deu suporte à hipótese 1. Também foi observada uma diferença significante ente os grupos rock e controle $(\mathrm{p}<0,05)$. Como os escores para a criatividade foram maiores em função da exposição ao rock, observou-se o suporte à hipótese 2 . Por outro lado, a diferença entre Mozart e rock não foi significante $(\mathrm{p}>0,05)$, o que deu suporte à hipótese 3.

Conforme mencionado, o teste não paramétrico de Mann-Whitney foi utilizado com vistas à verificação desses resultados. O teste evidenciou diferenças significantes entre os grupos controle e Mozart $(\mathrm{U}=387,00 ; \mathrm{z}=-3,23, \mathrm{p}<0,01)$ e entre controle e rock $(\mathrm{U}=370,50, \mathrm{z}=-2,23, \mathrm{p}<0,05)$. Novamente, não houve diferença significante entre Mozart e rock $(\mathrm{U}=1343,50, \mathrm{z}=-0,87, \mathrm{p}>0,05)$. Verifica-se que os resultados das comparações entre os grupos são equivalentes aos obtidos pela ANOVA, evidenciando a consistência entre os resultados dos testes.

\section{Discussão}

As três hipóteses foram suportadas pela análise Escore e, também, pela análise TopEscore, que envolve a indicação das respostas mais criativas pelos próprios respondentes. A análise TopEscore apresenta vantagens na avaliação de diferenças individuais quanto à criatividade, ao contornar a confusão entre originalidade e fluência; o método possui a vantagem de evitar que indivíduos que apresentam um número maior de respostas, sendo uma parte delas menos criativas, obtenham escores médios menores, sendo penalizados em relação a participantes que enumeram poucas respostas.

Observa-se que tanto a estimulação por Mozart como pelo rock se associaram a maiores níveis de pensamento divergente dos sujeitos e que não houve diferenças significantes entre esses dois estímulos. Desse modo, constata-se, novamente, um efeito da música sobre o desempenho cognitivo (neste estudo, a criatividade), encontrado em pesquisas anteriores: tanto por meio de Mozart, tal como nos trabalhos pioneiros de Rauscher et al. (1993), como pela exposição a outros gêneros musicais, tal como no experimento com música pop inglesa (Schellenberg \& Hallam, 2005).

É importante notar que o trecho de Mozart apresenta tempo acelerado e tonalidade maior, o que pode potencializar o aumento da excitação e mudanças de humor dos participantes, conforme o enfoque de Thompson et al. (2001). O segundo gênero examinado (rock) foi escolhido por apresentar características análogas em termos de tempo e tom (e também os efeitos em humor e excitação), tal como constatado por Schellenberg e Hallam (2005). Os resultados para os dois estilos musicais, tomados em conjunto, parecem reforçar os pressupostos de que o efeito cognitivo da música é promovido por meio da excitação e alteração de humor do ouvinte (Schellenberg \& Hallam, 2005; Thompson et al., 2001). Chabris (1999), baseado em uma meta-análise com 16 estudos, reforça que os efeitos transitórios nas habilidades dos sujeitos se relacionam ao humor e excitação induzidos pela música. Ele coloca que isso explica a dificuldade de alguns estudos alcançarem os mesmos efeitos com estímulos que não tinham o mesmo impacto emocional. De fato, deve-se observar que o grupo controle deste estudo, que não foi exposto aos 
estímulos musicais, apresentou menores resultados na avaliação de pensamento divergente dos que os demais grupos.

Outros estudos também reforçam tais pressupostos. Por exemplo, há pesquisas que indicam que há uma relação entre tonalidades maiores e andamentos mais rápidos com a manifestação de emoções positivas (Sutton \& Lowis, 2008; Webster \& Weir, 2005) e que a música, de fato, ativa áreas do cérebro ligadas às emoções (Särkämö et al., 2008). Sutton e Lowis (2008), por exemplo, testaram o efeito de um trecho de Hendel em Fá maior e de uma versão manipulada em tom menor; os sujeitos avaliaram o impacto emocional percebido, sendo que os resultados para a primeira versão indicaram mais efeitos emocionais positivos.

Além disso, Witvliet e Vrana (2007) identificaram que, na exposição a músicas que induzem excitação positiva, os sujeitos não só reportaram terem apreciado a experiência musical, como também emitiram reações fisiológicas mensuráveis - tal como a ativação dos músculos envolvidos no sorriso sugerindo que os sujeitos responderam emocionalmente e afetivamente à estimulação musical. Por outro lado, Isen, Dubman e Novicki (1987) constataram, por meio de um conjunto de experimentos, que a emergência de afetos positivos estimula a solução criativa de problemas. Assim, os autores sugerem que, em diferentes ambientes (educacional, organizacional, etc.), a intensificação de emoções positivas pode facilitar a expressão da criatividade. Também Baas, De Dreu e Nijstad (2008) identificaram que situações que induzem humores positivos (ex.: alegria) podem estimular maior criatividade do que situações envolvendo humor neutro.

Esses aspectos ajudam a compreender a mecânica do efeito da estimulação por meio da música e os seus efeitos cognitivos: aspectos emocionais parecem mediar esse processo. Por outro lado, como ponderam Schellenber e Hussain (2005) e Schellenberg, Nakata, Hunter e Tamoto (2007), diferentes gêneros musicais também podem promover tais efeitos. E mais: não é somente a música que pode influenciar o desempenho cognitivo (o que inclui a criatividade), outros estímulos que influenciam o estado emocional também têm potencial para isso (Schellenberg \& Weiss, 2013). Assim, a análise dos efeitos de outros estímulos cognitivos sobre o pensamento divergente parece ser um campo inspirador para futuros estudos, principalmente relacionados à criatividade nas organizações.

Algumas limitações deste estudo devem ser mencionadas, de modo a que possa colocar os presentes resultados em perspectiva e que novos estudos possam vir a gerenciar esses pontos. Primeiro, em função do tamanho reduzido da amostra e do fato de ela não ser randômica, os resultados não são passíveis de generalização. Segundo, por se tratar de um estudo cross-sectional, não se pode afirmar como seria o efeito dos estímulos musicais adotados sobre a criatividade, com o passar do tempo. Contudo, com base nos estudos anteriores envolvendo processos cognitivos, é de se esperar que tal efeito seja de curto prazo, durando de 10 a 15 minutos após a exposição ao estímulo (Nantais \& Schellenberg, 1999); esse aspecto pode ser explorado em futuros estudos.

Além disso, este estudo não apurou o efeito combinado da estimulação cognitiva com atributos pessoais (por exemplo: personalidade ou o perfil criativo dos participantes). Assim, no futuro, poderia ser investigado o efeito moderador de características individuais. Também poderiam ser utilizados diferentes estímulos musicais e estímulos não musicais. Ainda, uma interessante análise de criatividade no longo prazo seria replicar o teste de pensamento divergente segmentando grupos de músicos profissionais, músicos amadores e não músicos.

Por outro lado, uma vez que a influência da música também depende das preferências, apreciação e prazer do ouvinte com relação ao tipo de música experimentada (Nantais \& Schellenberg, 1999; Schellenberg \& Hallam, 2005), é possível que o rock contemporâneo escolhido não tenha o mesmo efeito em grupos com faixas etárias distintas à da amostra (entre 18 e 26 anos). Como esta música parece ser de maior interesse para o perfil dos estudantes envolvidos neste estudo, outros tipos musicais poderiam ser estudados junto a outros perfis de participantes, em novos estudos.

Finalmente, é interessante observar que a exposição continuada e regular à música, desde a infância, pode estimular incrementos duradouros em habilidades cognitivas (Schellenberg, 2005; 
Schellenberg et al., 2007). De fato, testes realizados com ressonância magnética apresentam evidência empírica de que há diferenças entre os cérebros de músicos e não músicos, e que essas diferenças se estendem para áreas não necessariamente ligadas à música, mas também ao aprendizado (Hyde et al., 2009). Novos estudos poderiam abordar o efeito da exposição regular à música sobre a criatividade de profissionais e gestores, no longo prazo.

\section{Considerações Finais}

Os resultados deste estudo reforçam as conclusões de estudos anteriores que apontam que a estimulação cognitiva pode influenciar processos cognitivos e contribuir com a ativação da criatividade. De fato, observa-se que estímulos ambientais que induzem tais variações nos estados de humor se desdobram em ideias e respostas mais criativas, caracterizadas - conforme a avaliação realizada - por serem mais incomuns, inteligentes e com associações mais fora dos padrões (Silvia et al., 2008).

Estes resultados contribuem com a literatura sobre criatividade nas organizações de duas maneiras: ao resgatar a relevância da estimulação cognitiva para o tema e ao chamar a atenção para as possíveis contribuições das ciências cognitivas e neurociências. No primeiro aspecto, percebe-se um gap nos estudos sobre criatividade nas organizações, que não têm explorado a estimulação cognitiva como antecedente (Anderson et al., 2014). Por outro lado, embora as ciências cognitivas e neurociências ainda sejam pouco exploradas em pesquisas sobre as pessoas nas empresas, trata-se de um campo que tem o potencial de contribuir com perspectivas multidisciplinares sobre o tema.

Outra contribuição deste estudo refere-se à metodologia utilizada, que explora a possibilidade de utilização de uma abordagem alternativa para a mensuração da criatividade. Trata-se de uma abordagem que contorna limitações associadas às medidas de criatividade usualmente empregadas, que envolvem pontuação das respostas singulares; estudos anteriores têm indicado resultados satisfatórios com relação à sua validade e confiabilidade (Benedek et al., 2013; Silvia et al., 2008). Neste trabalho, o método subjetivo adotado também apresentou índices de confiabilidade adequados; recomenda-se, contudo, que a sua validade e confiabilidade sejam examinados de modo mais amplo, para o contexto do nosso país.

Também seria adequado testar os efeitos específicos de cada tipo de tarefa de pensamento divergente (usos incomuns, instâncias e consequências). Sugere-se, então, que futuros trabalhos examinem esses aspectos, dado o potencial da metodologia para as pesquisas sobre pensamento divergente nas organizações. Alguns estudos têm apontado o modo como, na prática, a música poderia impactar as organizações. Por exemplo, o estudo de Lesiuk (2005) indicou que ouvir música está associado a incrementos na qualidade do trabalho e à ampliação da percepção - a criação de relações, o ver o todo, articulando múltiplas variáveis simultaneamente - no caso de desenvolvedores de softwares.

Outro trabalho indicou que a música no local trabalho pode melhorar o desempenho, promovendo inspiração, concentração, gestão do stress (permitindo à pessoa, alternadamente, engajar-se e escapar do trabalho) (Haake, 2011). Como foi observado neste artigo, as evidências sugerem que a música também tem o potencial de estimular a emergência do pensamento divergente, que é um processo central na geração de novas ideias e soluções; seria interessante investigar esse efeito também no setting organizacional (e implicações práticas), em futuras pesquisas.

\section{Referências}

Acar, S., \& Runco, M. (2012). Creative abilities: divergent thinking. In M. Mumford (Ed.), Handbook of organizational creativity (pp. 115-139). San Diego: Elsevier. 
Alencar, E., \& Fleith, D. (2010). A questão da medida em criatividade. In L. Pasquali (Org.), Instrumentação psicológica: fundamentos e práticas (pp. 324-341). Porto Alegre: Artmed.

Amabile, T. (1996). Creativity and innovation in organizations. Cambridge, MA: Perseus Publishing.

Amabile, T., Barsade, S. G., Mueller, J. S., \& Staw, B. M. (2005). Affect and creativity at work. Administrative Science Quarterly, 50(3), 367-403. doi: 10.2189/asqu.2005.50.3.367

American Psychological Association. (2002). Ethical principles of psychologists and code of conduct. American Psychologist Association, 57(12), 1060-1073.

Anderson, N., Potočnik, P., \& Zhou, J. (2014). Innovation and creativity in organizations: a state-ofthe-science review, prospective commentary, and guiding framework. Journal of Management, 40(5), 1297-1333. doi: 10.1177/0149206314527128

Atwater, L., \& Carmeli, A. (2009). Leader-member exchange, feelings of energy, and involvement in creative work. The Leadership Quarterly, 20(3), 264-275. doi: 10.1016/j.leaqua.2007.07.009

Baas, M., De Dreu, C. K., \& Nijstad, B. A. (2008). A meta-analysis of 25 years of mood-creativity research: hedonic tone, activation, or regulatory focus? Psychological Bulletin, 134(6), 779-806. doi: $10.1037 / \mathrm{a} 0012815$

Benedek, M., Mühlmann, C., Jauk, E., \& Neubauer, A. C. (2013). Assessment of divergent thinking by means of the subjective top-scoring method: effects of the number of top-ideas and time-on-task on reliability and validity. Psychology of Aesthetics, Creativity, and the Arts, 7(4), 341-349. doi 10.1037/a0033644

Blood, A., \& Zatorre, R. (2001). Intensely pleasurable responses to music correlate with activity in brain regions implicated in reward and emotion. Proceedings of the National Academy of Sciences of the United States of America, 98(20), 11818-11823. doi: 10.1038/7299

Chabris, C. F. (1999). Prelude or requiem for the "Mozart Effect"? Nature, 400, 826-827. doi: $10.1038 / 23608$

Christensen, P. R., Guilford, J. P., Merrifield, P. R., \& Wilson, R. C. (1960). Alternate uses. Beverly Hills, CA: Sheridan Psychological Services.

Christensen, P. R., Merrifield, P. R., \& Guilford, J. P. (1958). Consequences. Beverly Hills, CA: Sheridan Psychological Services.

Cronbach, J., Gleser, G., Nanda, H., \& Rajaratman, N. (1972). The dependability of behavioral measurements: theory of generalizability for scorers and profiles. New York, NY: John Wiley and Sons.

Davis, M. A. (2009). Understanding the relationship between mood and creativity: a meta-analysis. Organizational Behavior and Human Decision Processes, 108(1), 25-38. doi: 10.1016/j.obhdp.2008.04.001

Dul, J., \& Ceylan, C. (2014). The impact of a creativity-supporting work environment on a firm's product innovation performance. Journal of Product Innovation Management, 31(6), 1254-1267. doi: 10.1111/jpim. 12149

Fink, A., Grabner, R. H., Gebauer, D., Reishofer, G., Koschutnig, K., \& Ebner, F. (2010). Enhancing creativity by means of cognitive stimulation: evidence from an fMRI study. NeuroImage, 52(4), 1687-1695. doi: 10.1016/j.neuroimage.2010.05.072

Gaser, C., \& Schlaug, G. (2003). Brain structures differ between musicians and non-musicians. The Journal of Neuroscience, 23(27), 9240-9245. doi: 10.1523/JNEUROSCI.3403-13.2013 
Gibson, C., Folley, B. S., \& Park, S. (2009). Enhanced divergent thinking and creativity in musicians: a behavioral and near-infrared spectroscopy study. Brain and Cognition, 69(1), 162-169. doi: 10.1016/j.bandc.2008.07.009

Gilhooly, K. J., Fioratou, E., Anthony, S. H., \& Wynn, V. (2007). Divergent thinking: strategies and executive involvement in generating novel uses for familiar objects. British Journal of Psychology, 98(4), 611-625. doi: 10.1111/j.2044-8295.2007.tb00467.x

Haake, A. B. (2011). Individual music listening in workplace settings: an exploratory survey of offices in the UK. Musicae Scientiae, 15(1), 107-129. doi: 10.1177/1029864911398065

Hair, J., Black, W., Babin, B., \& Anderson, R. (2010). Multilevel data analysis: a global perspective. Upper Saddle River, NJ: Pearson Prentice Hall.

Hyde, K. L., Lerch, J., Norton, A., Forgeard, M., Winner, E., Evans, A. C., \& Schlaug, G. (2009). The effects of musical training on structural brain development: a longitudinal study. Annals of the New York Academy of Sciences, 1169, 182-186. doi: 10.1111/j.1749-6632.2009.04852.x

Im, S., Montoya, M., \& Workman, J. P., Jr. (2013). Antecedents and consequences of creativity in product innovation teams. Journal of Product Innovation Management, 30(1), 170-185. doi: 10.1111/j.1540-5885.2012.00887.x

Isen, A. M., Daubman, K. A., \& Nowicki, G. P. (1987). Positive affect facilitates creative problem solving. Journal of Personality and Social Psychology, 52(6), 1122-1131. doi: 10.1037/00223514.52.6.1122

Jausovec, N., \& Habe, K. (2005). The influence of Mozart's sonata K.488 on brain activity during the performance of spatial rotation and numerical tasks. Brain Topography, 17(4), 207-218. doi: $10.1007 / \mathrm{s} 10548-005-6030-4$

Koelsch, S. (2014). Brain correlates of music-evoked emotions. Nature Reviews Neuroscience, 15(3), 170-180. doi: 10.1038/nrn3666

Kuester, G., Rios, L., Ortiz, A., \& Miranda, M. (2010). Effect of music on the recovery of a patient with refractory nonconvulsive status epilepticus. Epilepsy \& Behavior, 18(4), 491-493. doi: 10.1016/j.yebeh.2010.06.001

Leng, X., \& Shaw, G. (1991). Toward a neutral theory of higher brain function using music as a window. Concepts in Neuroscience, 2(2), 229-258.

Lesiuk, T. (2005). The effect of music listening on work performance. Psychology of Music, 33(2), 173191. doi: $10.1177 / 0305735605050650$

Litchfield, R. C. (2008). Brainstorming reconsidered: a goal-based view. Academy of Management Review, 33(3), 649-668. doi: 10.5465/AMR.2008.32465708

McDonald, P., \& Tang, Y.-Y. (2014). Neuroscientific insights into management development: Theoretical propositions and practical implications. Group \& Organization Management, 39(5), 475-503. doi: 10.1177/1059601114550712

Moon, B.-J. (2013). Antecedents and outcomes of strategic thinking. Journal of Business Research, 66(10), 1698-1708. doi: 10.1016/j.jbusres.2012.11.006

Mushquash, C., \& O'Connor, B. P. (2006). SPSS and SAS programs for generalizability theory analyses. Behavior Research Methods, 38(3), 542-547. doi: 10.3758/BF03192810

Nantais, K. M., \& Schellenberg, E. G. (1999). The Mozart effect: an artifact of preference. Psychological Science, 10(4), 370-373. doi: 10.1111/1467-9280.00170 
Obendorf, H. (2009). Minimalism: designing simplicity. London: Springer-Verlag London Limited.

Pavlygina, R. A., Karamysheva, N. N., Tutushkina, M. V., Sakharov, D. S., \& Davydov, V. I. (2013). Solution of mathematical logical problems in a sensory enriched environment (classical music). Neuroscience and Behavioral Physiology, 43(6), 674-681. doi: 10.1007/s11055-013-9791-3

Peretz, I., \& Zatorre, R. J. (2005). Brain organization for music processing. Annual Review of Psychology, 56, 89-114. doi: 10.1146/annurev.psych.56.091103.070225

Plucker, J. A., \& Renzulli, J. S. (1999). Psychometric approaches to the study of human creativity. In R. Sternberg (Ed.), Handbook of creativity (pp. 35-61). Cambridge, UK: Cambridge University Press.

Popescu, M., Otsuka, A., \& Ioannides, A. A. (2004). Dynamics of brain activity in motor and frontal cortical areas during music listening: a magnetoencephalographic study. Neuroimage, 21(4), 1622-1638. doi: 10.1016/j.neuroimage.2003.11.002

Prichard, C., Korczynski, M., \& Elmes, M. (2007). Music at work: an introduction. Group \& Organization Management, 32(1), 4-21. doi: 10.1177/1059601106294485

Rauscher, F. H., Robinson, K. D., \& Jens, J. J. (1998). Improved maze learning through early music exposure in rats. Neurological Research, 20(5), 427-432.

Rauscher, F. H., \& Shaw, G. L. (1998). Key components of the Mozart effect. Perceptual and Motor Skills, 86(3), 835-841. doi: 10.2466/pms.1998.86.3.835

Rauscher, F. H., Shaw, G. L., \& Ky, N. K. (1993). Music and spatial task performance. Nature, 365, 611. doi: $10.1038 / 365611 \mathrm{a} 0$

Rauscher, F. H., Shaw, G. L., \& Ky, K. N. (1995). Listening to Mozart enhances spatial-temporal reasoning: towards a neurophysiological basis. Neuroscience Letters, 185(1), 44-47. doi: 10.1016/0304-3940(94)11221-4

Runco, M. A., \& Acar, S. (2012). Divergent thinking as an indicator of creative potential. Creativity Research Journal, 24(1), 66-75. doi: 10.1080/10400419.2012.652929

Särkämö, T., Tervaniemi, M., Laitinen, S., Forsblom, A., Soinila, S., Mikkonen, M., Autti, T., Silvennoinen, H. M., Erkkilä, J., Laine, M., Peretz, I., \& Hietanen, M. (2008). Music listening enhances cognitive recovery and mood after middle cerebral artery stroke. Brain, 131(3), 866876. doi: 10.1093/brain/awn013

Schellenberg, E. G. (2005). Music and cognitive abilities. Current Directions in Psychological Science, 14(6), 317-320. doi: 10.1111/j.0963-7214.2005.00389.x

Schellenberg, E. G., \& Hallam, S. (2005). Music listening and cognitive abilities in 10- and 11-yearolds: the blur effect. Annals of the New York Academy of Sciences, 1060, 202-209.

Schellenberg, E. G., Nakata, T., Hunter, P. G., \& Tamoto, S. (2007). Exposure to music and cognitive performance: tests of children and adults. Psychology of Music, 35(1), 5-19. doi: $10.1177 / 0305735607068885$

Schellenberg, E. G., \& Weiss, M. W. (2013). Music and cognitive abilities. In D. Deutsch (Ed.), The psychology of music (pp. 499-550). Amsterdam: Elsevier.

Schmithorst, V. J., \& Holland, S. K. (2003). The effect of musical training on music processing: a functional magnetic resonance imaging study in humans. Neuroscience Letters, 348(2), 65-68. doi: 10.1016/S0304-3940(03)00714-6 
Siegel, S., \& Castellan, N. (1988). Non-parametric statistics for the behavioral sciences. New York: McGraw Hill.

Silvia, P. J. (2011). Subjective scoring of divergent thinking: examining the reliability of unusual uses, instances, and consequences tasks. Thinking Skills and Creativity, 6(1), 24-30. doi: 10.1016/j.tsc.2010.06.001

Silvia, P. J., Martin, C., \& Nusbaum, E. C. (2009). A snapshot of creativity: evaluating a quick and simple method for assessing divergent thinking. Thinking Skills and Creativity, 4(2), 79-85. doi: 10.1016/j.tsc.2009.06.005

Silvia, P. J., Nusbaum, E. C., Berg, C., Martin, C., \& O’Connor, A. (2009). Openness to experience, plasticity, and creativity: exploring lower-order, high-order, and interactive effects. Journal of Research in Personality, 43(6), 1087-1090. doi: 10.1016/j.jrp.2009.04.015

Silvia, P. J., Winterstein, B. P., \& Willse, J. T. (2008). Rejoinder: the madness to our method: some thoughts on divergent thinking. Psychology of Aesthetics, Creativity, and the Arts, 2(2), 19313896. doi: 10.1037/1931-3896.2.2.109

Silvia, P. J., Winterstein, B. P., Willse, J. T., Barona, C. M., Cram, J. T., Hess, K. I., Martinez, J. L., \& Richard, C. A. (2008). Assessing creativity with divergent thinking tasks: exploring the reliability and validity of new subjective scoring methods. Psychology of Aesthetics, Creativity, and the Arts, 2(2), 68-85. doi: 10.1037/1931-3896.2.2.68

Steele, K. M., Bass, K. E., \& Crook, M. D. (1999). The mystery of the Mozart effect: failure to replicate. Psychological Science, 10(4), 366-369. doi: 10.1111/1467-9280.00169

Styhre, A. (2013). Sound, silence, music: organizing audible work settings. Culture and Organization, 19(1), 22-41. doi: 10.1080/14759551.2011.634197

Sutton, C. J. C., \& Lowis, M. J. (2008). The effect of musical mode on verbal and spatial task performance. Creativity Research Journal, 20(4), 420-426.doi: 10.1080/10400410802391884

Thompson, W. F., Schellenberg, E. G., \& Husain, G. (2001). Arousal, mood, and the Mozart effect. Psychological Science, 12(3), 248-251. doi: 10.1111/1467-9280.00345

Torrance, E. P. (2008). Torrance tests of creative thinking: norms-technical manual, verbal forms A \& $B$. Benseville, IL: Scholastic Testing Service.

Webster, G. D., \& Weir, C. G. (2005). Emotional responses to music: interactive effects of mode, texture, and tempo. Motivation and Emotion, 29(1), 19-39. doi: 10.1007/s11031-005-4414-0

Wei, D., Yang, J., Li, W., Wang, K., Zhang, Q., \& Qiu, J. (2014). Increased resting functional connectivity of the medial prefrontal cortex in creativity by means of cognitive stimulation. Cortex, 51, 92-102. doi: 10.1016/j.cortex.2013.09.004

Weinberger, N. M. (2004). Music and the brain. Scientific American, 291(5), 89-95.

Wilson, R. C., Guilford, J. P., \& Christensen, P. R. (1953). The measurement of individual differences in originality. Psychological Bulletin, 50(5), 362-370. doi: 10.1037/h0060857

Witvliet, C. V. O., \& Vrana, S. R. (2007). Play it again sam: repeated exposure to emotionally evocative music polarises liking and smiling responses, and influences other affective reports, facial EMG, and heart rate. Cognition and Emotion, 21(1), 3-25. doi: 10.1080/02699930601000672

Woodman, R. W., Sawyer, J. E., \& Griffin, R. W. (1993). Toward a theory of organizational creativity. Academy of Management Review, 18(2), 293-321. doi: 10.5465/AMR.1993.3997517 


\section{Dados dos Autores}

Guilherme Macedo de Souza Tieppo

Rua Itapeva, 474, 01332-000, São Paulo, SP, Brasil. E-mail: guilherme.tieppo@gmail.com

Germano Glufke Reis

Rua Taguá, 150, Liberdade, 01508-010, São Paulo, SP, Brasil. E-mail: germano.reis@fgv.br

Djair Picchiai

Rua Itapeva, 474, 01332-000, São Paulo, SP, Brasil. E-mail: djair.picchiai@fgv.br 